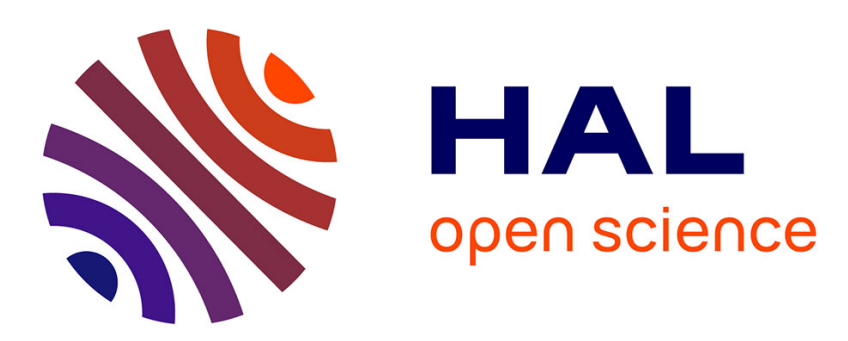

\title{
A prospective, randomized study on the efficacy of tongue protector in patients with burning mouth syndrome
}

Pía López-Jornet, Fabio Camacho-Alonso, M Paz Andujar-Mateos

\section{- To cite this version:}

Pía López-Jornet, Fabio Camacho-Alonso, M Paz Andujar-Mateos. A prospective, randomized study on the efficacy of tongue protector in patients with burning mouth syndrome. Oral Diseases, 2010, 17 (3), pp.277. 10.1111/j.1601-0825.2010.01737.x . hal-00599895

\section{HAL Id: hal-00599895 https://hal.science/hal-00599895}

Submitted on 11 Jun 2011

HAL is a multi-disciplinary open access archive for the deposit and dissemination of scientific research documents, whether they are published or not. The documents may come from teaching and research institutions in France or abroad, or from public or private research centers.
L'archive ouverte pluridisciplinaire HAL, est destinée au dépôt et à la diffusion de documents scientifiques de niveau recherche, publiés ou non, émanant des établissements d'enseignement et de recherche français ou étrangers, des laboratoires publics ou privés. 


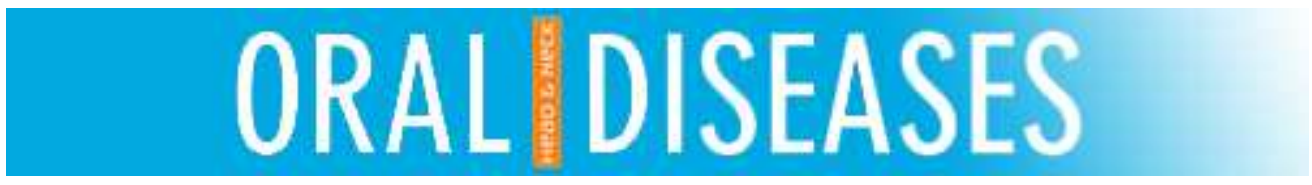

\section{A prospective, randomized study on the efficacy of tongue protector in patients with burning mouth syndrome}

\begin{tabular}{|r|l|}
\hline Journal: & Oral Diseases \\
\hline Manuscript ID: & ODI-05-10-OM-1669.R2 \\
\hline Manuscript Type: & Original Manuscript \\
\hline Date Submitted by the & $16-J u n-2010$ \\
\hline Author: & $\begin{array}{l}\text { Complete List of Authors: } \\
\text { de Murcia, Oral Medicine } \\
\text { Andujar-Mateos, M Paz; University Murcia }\end{array}$ \\
\hline Keywords: & Medicine, Genetics \\
\hline
\end{tabular}

\section{今scholarONE* Manuscript Central}


Title: A prospective, randomized study on the efficacy of tongue protector in patients with burning mouth syndrome

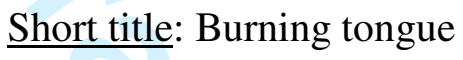

Original Article type

Key words: Burning mouth syndrome; primary; secondary; parafunctional habits; tongue; treatment.

Authors: Pia López-Jornet, Fabio Camacho-Alonso, Paz Andujar-Mateos Department of Oral Medicine. University of Murcia. Murcia (Spain)

Correspondence:

Pía López-Jornet

Clínica Odontológica Universitaria. Medicina Bucal

Hospital Morales Meseguer

Avda. Marqués de los Vélez s/n

30008 - Murcia (Spain)

Phone: 0034968398588

Fax: 0034968398576

E-mail: majornet@um. es 


\section{ABSTRACT}

Objective: was to apply a tongue protector with habit-modifying therapy through selfcontrol, in the patients with burning mouth syndrome (BMS).

Methods: A prospective, randomized study was made of 65 consecutive patients with BMS. Fifty subjects were randomized to two groups: group A (informed) and group B (informed and the application of a tongue protector). The symptoms were evaluated by VAS, while the psychological profile was assessed using the HAD, with application of the quality of life questionnaires SF-36 and OHIP-49. The duration of treatment was two months.

Results: Fifty patients (46 females and 4 males) completed the study. The VAS scores in group B were 8.2 at baseline and 4.5 after two months. The respective scores in group A were 7.1 and 5.6 - the differences between the two groups being significant $(\mathrm{p}<0.001)$. In group B the OHIP-49 yielded lower scores for most of the scales, with significant differences versus group A. In group B the SF 36 yielded significant differences versus group A in physical role, bodily pain, general health and emotional role.

Conclusions: Parafunctional traumatism of the tongue should be taken into account in the pathogenesis of BMS with a view to exploring new therapeutic options.

Key words: Burning mouth syndrome; primary; secondary; parafunctional habits; tongue; treatment. 


\section{INTRODUCTION}

Burning mouth syndrome (BMS) is characterized by a subjective sensation of burning or itching of the oral mucosa, in the absence of clinical and laboratory test data capable of accounting for the symptoms, and with a duration of at least 4-6 months (Grushka et al., 2006; Klasser et al., 2008; Fedele et al., 2007). The prevalence of BMS varies between 0.7-4.5\% (Scala et al., 2203; Maltsman et al., 2007). It is mainly seen in postmenopausal women (Baker et al., 2005). There is debate in the medical literature regarding the etiopathogenesis of the disorder, and several factors and concepts have been proposed (Scala et al, 2003; Lamey 1998; Bergdahl et al, 1995; Patton et al, 2007; Danhauer et al, 2002; Rojo et al, 1994; López-Jornet et al, 2008, Sardella et al, 2006; Zakrzewska et al, 2003). The postulated causal factors have been grouped into local factors (infections, irritants, etc.), systemic factors (diabetes mellitus, anemia through iron or folic acid deficiency, etc.), and psychogenic factors; but these causal factors contradict the definition of BMS and so constitute another disease condition separate from BMS Burning mouth syndrome is considered to be idiopathic/primary when the cause is impossible to determine (Klasser et al, 2008) and secondary when it is possible to identify etiologic factors for the syndrome. Some investigators have suggested that the disorder may be a manifestation of somatization, while others have reported it to be more closely related to neuropathic pain than to somatoform chronic pain syndromes. Parafunctional habits such as tongue thrusting or certain tics in the form of continuous rubbing of the teeth or dentures, lip, cheek or tongue nibbling, compulsive movements and hyperactivity of the tongue may contribute to induce and maintain the syndrome (Lopez-Jornet et al, 2009a). Pain in BMS is most often bilateral and symmetrical in the anterior two-thirds of the tongue (71-78\%), followed by the dorsal and lateral surfaces of the tongue, the anterior portion of the hard palate, and the lip mucosa and gingiva. The condition often manifests in several locations (Scala et al, 2003).

Considering the chronic nature of BMS and its prevalence, optimum treatment must be established, since no effective therapies have been developed to date. Many treatments have been tested with a view to securing symptoms relief, including benzodiazepines, tricyclic antidepressants, gabapentin, serotonin reuptake inhibitors, lipoic acid and cognitive behavioral therapy - with variable results. It is therefore important to study and understand the physiopathological mechanisms of stomatodynia in order to select the best possible treatment (Klasser et al, 2008;Maina et al, 2005; Minguez Serra et al, 2007; Gremeau Richard et al, 2004; Femiano et al, 2004; Lopez-Jornet et al, 2009 b; 
Carbone et al, 2009 ).

The proposed pharmacological protocols have not consistently proved to be predictable and effective in all BMS subjects, in this sense the aim of the present study was to apply a tongue protector with habit-modifying therapy through self-control, and to evaluate the symptoms and quality of life in the patients with burning mouth syndrome (BMS).

\section{METHODS}

A prospective, randomized study was made of 65 consecutive patients with BMS seen in the Department of Oral Medicine (University of Murcia, Spain). The study was carried out during the period between March 2007 and June 2009, and was authorized by the Bioethics Committee of the University of Murcia. A sample size was not calculated before starting the trial, as we were not able to predict the effect of the investigated treatment.

Inclusion criteria for participating in the study were a clinical history of continuous symptoms of oral burning or pain on a daily or almost daily basis, during all or part of the day for more than 6 months, without paroxysms, and independent of the nervous pathway. Likewise, the included patients presented no clinical abnormalities that could account for the symptoms. Furthermore, the patients had to present normal blood test findings (complete blood count, blood glucose, serum iron and transferrin levels, serum vitamin B12 and folate), and were required to provide informed consent (Gremeau Richard et al, 2004). Patients with pain attributable to other conditions (angiotensinconverting enzyme inhibitor use, candidiasis, lichenoid reactions, sores, tongue atrophy, etc.) were excluded, as were those presenting problems with dentures, biochemical anomalies and a history of hypersensitivity or allergy to the material used. Patients with known neurological disorders and those previously treated, even irregularly, with antidepressants, anticonvulsants, other psychotropic drugs, or psychological therapy were also excluded from the study. Patients occasionally using anxiolytics to induce sleep were accepted. Subjects with signs of lingual and labial parafunctional activity were also considered (tongue rubbing, lip or cheek nibbling) (Figure1).

Primary outcome variable: oral symptoms were registered using a visual analog scale (VAS) consisting of a $10-\mathrm{cm}$ vertical line marked from 0 (= no pain) to 10 (= most severe pain experienced) (Maina et al, 2005). Patients were asked to indicate the mean pain intensity for the two weeks preceding the consultation. The difference between baseline and the endpoint scores numerically expressed symptoms variation. 
The Hospital Anxiety and Depression scale (HAD) was used to evaluate the patient psychological profile. The HAD comprises two subscales relating to anxiety and depression. Each subscale contains 7 items pertaining to mood disorder. On analyzing the HAD scales, scores of over 10 indicated the probable presence of anxiety or depression, scores of 7 or less indicated no significant anxiety or depression, and scores of 8-10 were taken to be of borderline significance (Bjelland et al, 2002).

The Oral Health Impact Profile (OHIP-49) is an instrument used to measure oral health. With the OHIP-49, each item was scored as follows: 0 = "never", 1 = "hardly ever", $2=$ "occasionally", 3 = "fairly often", and 4 = "very often". The OHIP-49 is divided into 7 different domains, and the possible score range for each domain is: "functional limitation" (9 items), from 0-36; "physical pain" (9 items), from 0-36; "psychological discomfort" (5 items), from 0-20; "physical disability" (9 items), from 0-36; "psychological disability" (6 items), from 0-24; "social disability" (5 items), from 0-20; "handicap" (6 items), from 0-24; and finally "overall OHIP score" (49 items), from 0196. In this instrument, higher scores indicate a poorer state of health ( Lopez and Baelum 2006) .

The Short Form 36 (SF-36) Health Survey Questionnaire in turn has been designed to evaluate quality of life. The standard version of the SF-36 contains 8 areas (Alfonso et al., 1995): "physical functioning", "physical role limitations", "bodily pain”, "general medical health", "vitality", "social functioning", "emotional role limitations", and "mental health". The scoring system is designed in such a way that higher scores indicate better health; thus, 0 is the worst state of health and 100 the ideal state of health.

Every new patient seen at the Oral Medicine Unit who met the eligibility criteria was asked to enter the trial. After being informed about the scope and methods of the study, the subjects who accepted signed the written informed consent form, and then were randomly allocated to one of the two arms of the study. The random allocation sequence was generated using software available online at http://graphpad.com/quickcalcs/randomize1. All the patients were instructed not to use any product for BMS during inclusion in the study.

All the patients in group A were informed in detail about their illness, and were instructed not to rub their tongue against their teeth and/or dentures. A self-control technique was used to this effect, the patients being given 10 printed habit-modifying 
reminder points to be placed in visible places. The same measures were adopted in group $\mathrm{B}$, though in this case the patients were moreover programmed for tongue protector placement. The protector consisted of a transparent, low-density polyethylene sheath covering the tongue from the tip to the posterior third. These tongue protector $\mathrm{s}$ were single-use devices measuring $0.1 \mathrm{~mm}$ in thickness, with a standard size $(67 \mathrm{~mm}$ in length and $66 \mathrm{~mm}$ wide), and were custom manufactured by our group (figure 2). Each patient received a kit with the protectors and the reminder points for treatment. Instructions were provided on their use - the protector being worn during the daytime for a period of two months. We recommended use of the protector 15 minutes / three times a day with the therapeutic aim of avoiding continuous rubbing against the teeth and/or dentures.

The patients in both groups were examined at the start of treatment and again after two months. One month after inclusion, the investigator called the patients in both groups by telephone as a reminder and to reinforce motivation. All patients completed the HAD questionnaire and the quality of life instruments OHIP 49 and SF-36 on each of the visits (after 0 and 2 months). On these visits the subjects were also questioned about tolerability and possible adverse effects.

The data were analyzed using the SPSS version 12.0 statistical package (SPSS ${ }^{\circledR}$ Inc., $^{-}$ Chicago, IL, USA). A descriptive study was made of each variable. The associations between the different qualitative variables were studied using Pearson's chi-squared test. We used the Student t-test for two independent samples in application to quantitative variables - in each case determining whether the variances were homogeneous. A probability of less than $\mathrm{p} \leq 0.05$ was accepted as significant.

\section{RESULTS}

Sixty-five patients referred to the Department of Oral Medicine for oral burning sensation were assessed for eligibility: 12 failed to meet the inclusion criteria and three refused to participate in the study. A total of 50 subjects were therefore finally included: 46 females and 4 males, aged 61.18 \pm 12.27 years (range 37-84). Table 1 shows the homogeneity of the two patient groups on the day of inclusion in the study. During the two-month duration of the study there were no dropouts in either group, and no adverse effects were observed.

The VAS scores in group B (tongue protector group) were 8.2 at baseline and 4.5 after two months. The respective scores in group A were 7.1 and 5.6) - the differences 
between the two groups being significant $(\mathrm{p}<0.001)$ (Table 2). Regarding the HAD, the scores showed no significant differences for either of the groups during the course of the study.

In group B the OHIP-49 (Table 3) yielded lower scores (i.e., better quality of life) in all domains, with significant differences between the two study time points (day 0 and after two months). Thus, the total OHIP-49 score in group B was $62.9 \pm 34.0$ on day 0 and $44.5 \pm 28.1$ after two months. In contrast, group A showed only minimum changes, with a total score of $55.4 \pm 4$ on day and $53.7 \pm 35.3$ after two months. On comparing the two patient groups, significant differences were observed in the scales or domains corresponding to functional limitation, bodily pain, psychological discomfort, physical disability, psychological disability, and total score.

Lastly, as refers to the SF-36 (Table 4), the patients subjected to tongue protector treatment (group B) showed improvement in the scores between day 0 and the end of treatment ( 2 months) in practically all the subscales, while only minimal changes were observed in group A. On comparing the two groups, significant differences were recorded for physical functioning, physical role, bodily pain, general health and emotional role (Table 4).

\section{DISCUSSION}

Our results indicate clinical improvement among the patients for burning mouth syndrome (BMS). The VAS scores were modified in both groups during the treatment period (0-2 months), though significant differences were observed between them. On only considering overall improvement, the mean reduction in VAS score observed in group B was from 8.2 at baseline to 4.5 at the end of the study. The mean 1.4-point decrease in pain intensity observed in this study in group A may appear small, but obviously all patients did not respond in the same way to the active treatment.

Burning mouth syndrome is a diagnostic and therapeutic challenge because of its etiopathogenic variability (Patton et al, 2007; Kasser et al, 2008). The latest studies suggest that psychopathological factors may play an important role in the disorder, in correlation with the multifactorial origin of BMS. A strong psychological component in BMS has been clearly identified in the last decade. It has been suggested that somatic complaints from unfavorable life experiences associated with chronic pain may influence both individual personality and mood changes. Bergdahl et al, 1995, studied the effect of cognitive therapy on resistant BMS. Their patients were randomly divided 
into two equal groups: a therapy group was subjected to cognitive therapy and an attention / placebo group served as control group. The authors used a visual analog scale to estimate the intensity of BMS, and they found it to be significantly reduced in the therapy group after cognitive therapy was completed (12-15 one-hour sessions once a week).

Gremeau-Richard et al, 2004, published a randomized double-blind, multicenter parallel group study in which topical clonazepam improved the stomatodynia symptoms (twothirds of the included subjects). However, this treatment was not effective in all subjects. Several studies have assessed systemic therapies, including amisulpiride, selective serotonin reuptake inhibitor antidepressants such as paroxetine and sertraline, trazodone and $0.25 \%$ capsaicin, with variable results (Patton et al, 2007; Minguez Serra et al. 2007). The use of lipoic acid in BMS patients was presented as a promising alternative for management of the symptoms, with high levels of improvement - though lipoic acid is not effective in many cases (Lopez Jornet et al, 2009b, Carbone et al, 2009).

Oral parafunctional habits have been widely implicated as factors that intervene in the development and perpetuation of other syndromes (Axel, 2008, Dworkin et al, 1992) such as for example temporomandibular joint disorders. Parafunctional activity is defined as the potential lesions (depending on the tolerance of the individual in question) caused by a series of movements that occur in parallel to normal function, though lacking a functional purpose, and which generate traumatic forces characterized by an abnormal direction, excessive intensity, and a frequent and lasting character. Any type of hyperactivity - in this case of the tongue and/or lips - occurring without functional objectives or in a way that proves inadequate for the stomatognathic system, is regarded as parafunctional activity. In this context, the tongue protector used in our study offered protection, since it avoided direct friction or rubbing of the tongue mucosa against the teeth and/or dentures protect the tongue regarding the changes in temperature and taste, salivary flow (increased) though its use could also exert a placebo effect.

The management of BMS symptoms is the greatest challenge related with this syndrome. Several systemic and local therapies have been suggested to treat these patients, with frequently unsatisfactory results. Maybe this fact explains the high receptivity of BMS patients for new therapies and the good adherence to treatment 
observed in our study.

A possible confounding factor is represented by occult candidiasis. Some authors reported evidence for candida-induced burning mouth even in the absence of objective signs (Terai and Shimahara, 2007), thus suggesting the advisability of routine treatment with antifungal agents for at least one week before confirming the diagnosis of BMS. As this issue was not taken into account in the present protocol, some patients may have had occult candidiasis, and therefore perhaps failed to respond to treatment.

Moreover, as the evaluation of symptoms could be altered by the interpersonal relationship between the patient and investigator, the same examiner followed-up on all of the patients in the study, in order to ensure that the results would be comparable.

It is important to consider quality of life in BMS patients, since the impact of the disease on the different domains or subscales is considerable. Accordingly, in our study use was made of two validated instruments, the OHIP-49 and SF-36. Our results show significant improvements in the OHIP-49 score in the group of patients treated with the tongue protector, in the domains relating to functional limitation, bodily pain, psychological discomfort, physical disability, psychological disability and in the total score.

As regards the SF-36, after two months of treatment group B showed improvement in all the domains, with significant differences versus group A as regards the subscales used to assess physical functioning, physical role, bodily pain, general health, and emotional role. Thus, the tongue protector could be an alternative for the management of patients with BMS. Self-inflicted damage secondary to parafunctional activity of the tongue should be taken into consideration in the pathogenesis of BMS, with a view to developing new management strategies.

Although our working hypothesis may be supported statistically, we included only a small patient series treated for a period of two months - a fact that makes it difficult to draw firm conclusions. Future studies are needed to reproduce our findings in larger series, over longer periods of time and involving an adequate sample of patients.

It is essential to deepen our understanding of the physiopathological mechanisms of burning mouth in order to select the best treatment and develop new therapies which may use different mechanisms. Future research moreover should focus on the long-term effects of treatment and on the quality of life of patients with burning mouth syndrome, in order to establish the clinical impact of such interventions 


\section{References}

Alonso J, Prieto L, Anto JM.(1995). La versión española del SF-36 Health Survey (Cuestionario de Salud SF-36): un instrumento para la medida de los resultados clínicos. Med Clin 104:771-776.

Axéll T. Treatment of smarting symptoms in the oral mucosa by appliance of lingual acrylic splints (2008). Swed Dent J 32:165-169.

Barker KE, Savage NW (2005). Burning mouth syndrome: an update on recent findings Aust Dent J 50:220-223.

Bergdahl J, Anneroth G, Perris H (1995). Cognitive therapy in the treatment of patients with resistant burning mouth syndrome: a controlled study. J Oral Pathol Med 24:213-215.

Bjelland I, Dhal A, Huag T, Neckelmann D (2002). The validity of the Hospital Anxiety and Depression Scale. An updated literature review. J Psychosom Res 2:69-77.

Carbone M, Pentenero M, Carrozzo M, Ippolito A, Gandolfo S (2009). Lack of efficacy of alpha-lipoic acid in burning mouth syndrome: a double-blind, randomized, placebo-controlled study. Eur J Pain 13:492-496.

Danhauer SC, Miller CS, Rhodus NL, Carlson CR (2002). Impact of criteria-based diagnosis of burning mouth syndrome on treatment outcome. J Orofac Pain 16:305-311.

Dworkin SF, LeResche L (1992). Research diagnostic criteria for temporomandibular disorders: review, criteria, examinations and specification, critique. J Craniomandib Disord Facial Oral Pain 6:301-355.

Fedele S, Fricchione G, Porter SR, Mignogna MD(2007). Burning mouth syndrome (stomatodynia). QJM 100:527-530.

Femiano F, Gombos F, Scully C. Síndrome de boca ardiente. Estudio de la psicoterapia, medicación con ácido alfa-lipoico y combinación de terapias. Med Oral 2004; 9:8-13.

Gremeau Richard C, Woda A, Navez ML, et al (2004). Topical clonazepam in stomatodynia: a randomized placebo-controlled study. Pain 188:51-57.

Grushka M, Ching V, Epstein J (2006). Burning mouth syndrome. Adv Otorhinolaryngol ; 63:278-287.

Klasser GD, Fischer DJ, Epstein JB(2008). Burning mouth syndrome: recognition, understanding, and management. Oral Maxillofac Surg Clin North Am 20:255-257. 
Lamey PJ (1998). Burning mouth syndrome: approach to successful management. Dent Update25:298300 .

López -Jornet P, Camacho Alonso F, Lucero Berdugo M (2008). Quality of life in patients with burning mouth syndrome. J Oral Pathol Med 37:389-394.

López-Jornet P, Camacho-Alonso F, Leon-Espinosa S (2009). Burning mouth syndrome, oral parafunctions, and psychological profile in a longitudinal case study. J Eur Acad Dermatol Venereol 23:363-365.

Lopez-Jornet P, Camacho- Alonso F, Leon-Espinosa S (2009). Efficacy of alpha lipoic acid in burning mouth syndrome: a randomized, placebo-treatment study J Oral Rehabil 36: 52-57.

Lopez R, Baelum V (2006). Spanish version of the Oral Health Impact Profile (OHIP-Sp). BMC Oral Health 6:1-8.

Maina G, Vitalucci A, Gandolfo S, Bogetto F (2002). Comparative efficacy of SSRIs and amisulpride in burning mouth syndrome: a single-blind study. J Clin Psychiatry 63:38-43.

Maina G, Albert U, Gandolfo S, Vitalucci A, Bogetto F(2005). Personality disorders in patients with burning mouth syndrome. J Personal Disord 19:84-89.

Maltsman-Tseikhin A, Moricca P, Niv D (2007). Burning mouth syndrome: will better understanding yield better management? Pain Pract 7: 151-162.

Minguez Serra MP, Salort Llorca C, Silvestre Donat FJ (2007). Pharmacological treatment of burning mouth syndrome: a review and update. Med Oral Patol Oral Cir Bucal 12: E299-304.

Patton LL, Siegel MA, Benoliel R, De Laat A. Management of burning mouth syndrome: systematic review and management recommendations. Oral Surg Oral Med Oral Pathol Oral Radiol Endod 103: e1-S39. e13.

Rojo L, Silvestre FJ, Bagan JV, de Vicente T.(1994) Prevalence of psychopathology in burning mouth syndrome. Oral Surg Oral Med Oral Pathol 78:312-316.

Sardella A, Lodi G, Demarosi F, Uglietti D, Carrassi A (2006). Causative or precipitating aspects of burning mouth syndrome: a case-control study. J Oral Pathol Med 35:466-471.

Scala A, Checchi L, Montevecchi M, Marini I, Giamberardino MA (2003). Update on burning mouth syndrome: Overview and patient management. Crit Rev Oral Biol Medical 14:275-291. 
Terai H, Shimahara M (2007). Tongue pain: burning mouth syndrome vs Candida associated lesion. Oral Dis 13:440-442

Zakrzewska JM, Forssell H, Glenny A (2003). Interventions for the treatment of burning mouth syndrome: A systematic review. J Orofacial Pain 17:293-300. 
Figure 1. Flow diagram

Figure 2. View of the tongue protector .Manufactured by our group with a standard size (67 $\mathrm{mm}$ in length and $66 \mathrm{~mm}$ wide) 


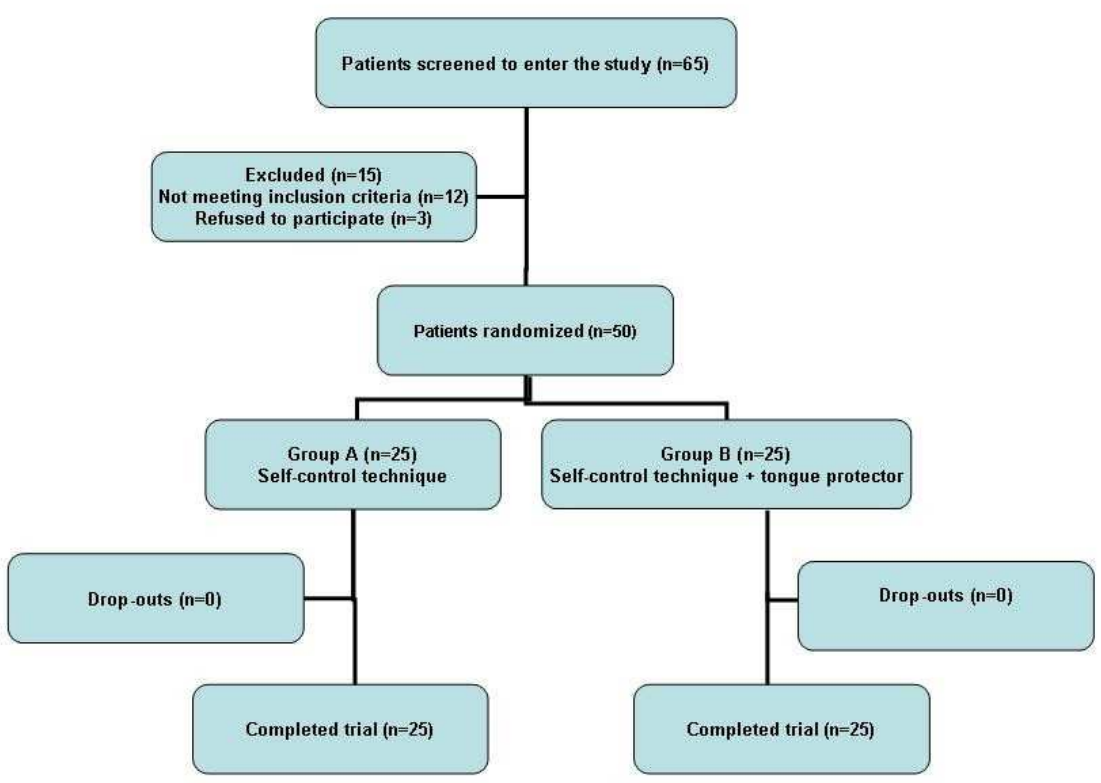

Fig. 1. Flow diagram of the trial

254x190mm (96 x 96 DPI) 


\section{Page 15 of 19}

Oral Diseases - Manuscript Copy

1

2

3

4

5

6

7

8

10

11

12

13

14

15

16

17

18

19

20

21

22

23

24

25

26

27

28

29

30

31

32

33

34

35

36

37

38

39

40

41

42

43

44

45

46

47

48

49

50

51

52

53

54

55

56

57

58

59

60

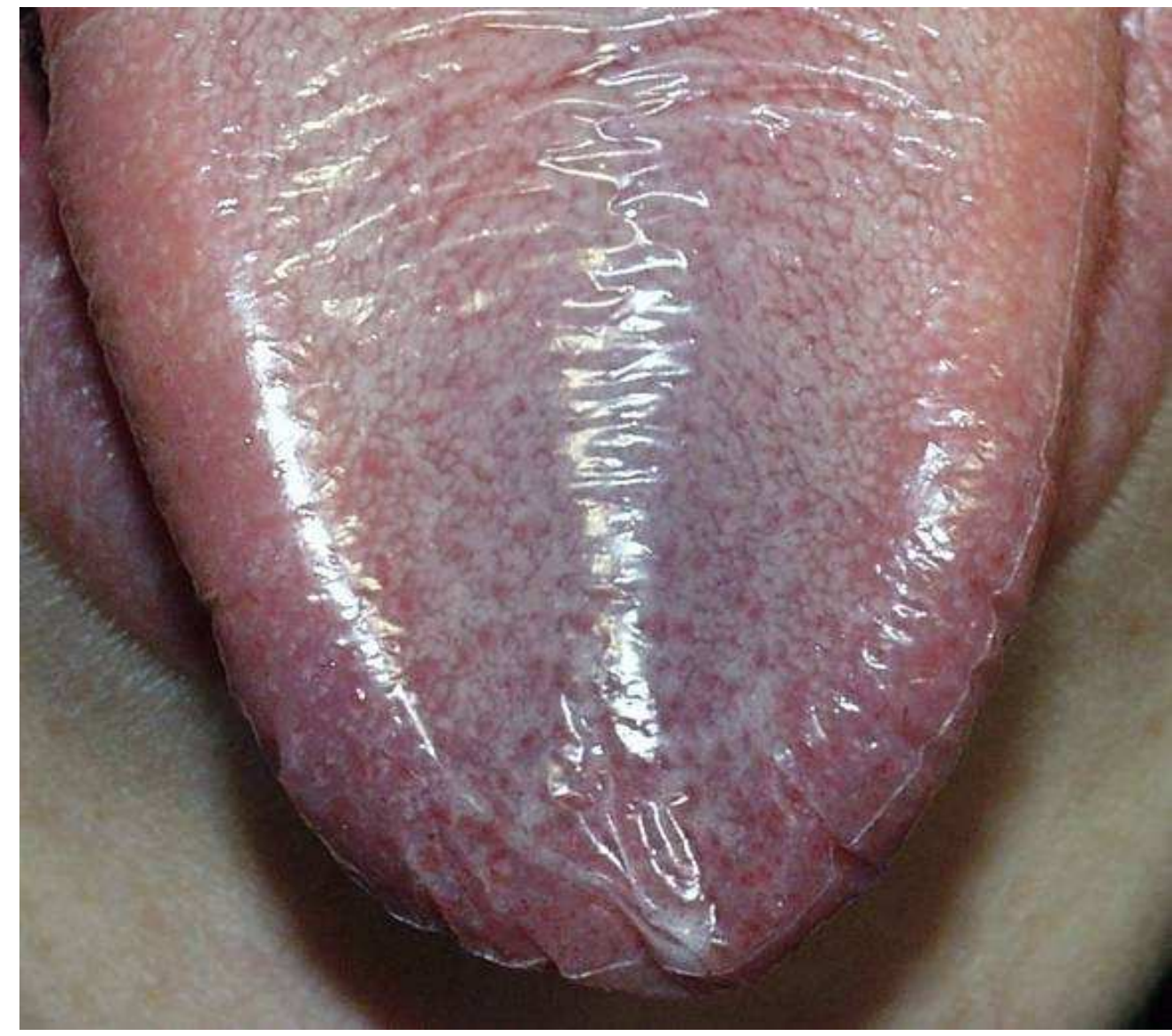

$98 \times 87 \mathrm{~mm}(150 \times 150 \mathrm{DPI})$ 
Table 1. Homogeneity of the study groups on day 0 (baseline) in relation to age, gender and the VAS and HAD scores (Student t-test and Pearson $\chi^{2}$ ).

\begin{tabular}{|c|c|c|c|}
\hline $\begin{array}{l}\text { Age, gender, VAS, HAD } \\
\text { day } 0\end{array}$ & $\begin{array}{c}\text { Group B } \\
(\mathbf{n}=\mathbf{2 5})\end{array}$ & $\begin{array}{c}\text { Group } A \\
(\mathbf{n}=25)\end{array}$ & P-value \\
\hline Age: mean \pm SD & $60.96 \pm 11.97$ & $61.40 \pm 12.81$ & 0.901 \\
\hline $\begin{array}{l}\text { Gender } \\
\text { Male: n }(\%) \\
\text { Female: n }(\%)\end{array}$ & $\begin{array}{l}2(8) \\
23(92)\end{array}$ & $\begin{array}{l}2(8) \\
23(92)\end{array}$ & 1.000 \\
\hline VAS: mean \pm SD & $8.2 \pm 2.1$ & $7.1 \pm 2.1$ & 0.087 \\
\hline $\begin{array}{l}\text { HAD: } \text { mean } \pm \text { SD } \\
\text { Depression } \\
\text { Anxiety }\end{array}$ & $\begin{array}{r}9.28 \pm 6.00 \\
11.12 \pm 5.27\end{array}$ & $\begin{array}{r}8.96 \pm 6.09 \\
11.24 \pm 5.67\end{array}$ & $\begin{array}{l}0.852 \\
0.939\end{array}$ \\
\hline
\end{tabular}


1 3 4 5 6$$
7
$$$$
8
$$$$
9
$$

10

Table 2. Evolution of the VAS and HAD scores during the two months of the study (Student t-test).

\begin{tabular}{|c|c|c|c|}
\hline \multirow{2}{*}{ VAS and HAD } & Group B $(n=25):$ mean \pm SD & Group A $(n=25):$ mean \pm SD & \multirow{2}{*}{ P-value } \\
\hline & Evolution (day 0 - 2 months) & Evolution (day 0 - 2 months) & \\
\hline VAS & $(8.2 \pm 2.1)-(4.5 \pm 2.2)=3.6 \pm 2.2$ & $(7.1 \pm 2.1)-(5.6 \pm 1.5)=1.4 \pm 1.6$ & $<0.001$ \\
\hline \multicolumn{4}{|l|}{ HAD } \\
\hline Depression & $(9.28 \pm 6.00)-(8.28 \pm 5.93)=1.00 \pm 3.73$ & $(8.96 \pm 6.09)-(8.92 \pm 6.13)=0.04 \pm 0.20$ & 0.205 \\
\hline Anxiety & $(11.12 \pm 5.27)-(11.20 \pm 6.59)=-0.08 \pm 3.39$ & $(11.24 \pm 5.67)-(11.04 \pm 5.43)=0.20 \pm 1.00$ & 0.694 \\
\hline
\end{tabular}

Note: $\mathrm{SD}=$ standard deviation; VAS = visual analog scale; HAD = Hospital Anxiety-Depression scale 
Table 3. Evolution of the OHIP-49 score during the two months of the study (Student t-test). 
2 3 4 6

8 9 Physical functioning 11 Physical role

Table 4. Evolution of the SF-36 score during the two months of the study (Student t-test).

12 13odily pain 14 15 General health 16 1 Nitality 18 19 20 21 Emotional role 22 23 Mental health 24 25 Vote: SD = standard deviation 